OPEN ACCESS

Edited by:

Claudio Pignata,

University of Naples Federico II, Italy

Reviewed by:

Lovro Lamot,

University of Zagreb, Croatia

Maria Vincenza Mastrolia,

University of Florence, Italy

*Correspondence:

Zhihui Li

Lizh0731@aliyun.com

tThese authors have contributed equally to this work and share first authorship

Specialty section:

This article was submitted to Pediatric Immunology,

a section of the journal

Frontiers in Pediatrics

Received: 19 March 2021 Accepted: 26 May 2021

Published: 18 June 2021

Citation:

Kang Z, Xu J and Li Z (2021) Juvenile Idiopathic Arthritis With Epstein-Barr Virus-Associated Smooth Muscle Tumor in a 6-Year-Old Girl: A Rare Case Report.

Front. Pediatr. 9:680113 doi: 10.3389/fped.2021.680113

\section{Juvenile Idiopathic Arthritis With Epstein-Barr Virus-Associated Smooth Muscle Tumor in a 6-Year-Old Girl: A Rare Case Report}

\author{
Zhijuan Kang ${ }^{1,2+}$, Juan $\mathrm{Xu}^{2 \dagger}$ and Zhihui $\mathrm{Li}^{1,2 *}$ \\ ${ }^{1}$ Department of Nephrology and Rheumatology of Hunan Children's Hospital, Changsha, China, ${ }^{2}$ Academy of Pediatrics of \\ University of South China, Changsha, China
}

Herein, we reported a rare case of Epstein-Barr virus-associated smooth muscle tumor (EBV-SMT) combined with juvenile idiopathic arthritis (JIA) in a 6-year old girl without HIV, organ transplantation, or congenital immunodeficiency. The patient suffered from pain in the bilateral hip joints, which drastically affected her physical activity. Consequently, she was diagnosed with JIA (September 2019). She was given methotrexate and methylprednisolone pills via oral route and a subcutaneous injection of Recombinant Human Tumor Necrosis Factor- $\alpha$ Receptor II;IgG Fc Fusion Protein for 4 weeks that successfully relieved the pain. In May 2020, the pain reoccurred and was accompanied by occasional headaches. After extensive pathological examination, the patient was diagnosed with EBV-SMT. The imaging examinations after admission showed multiple lesions in the skull, lungs, and vertebral body. Biopsy of the $\mathrm{L} 2$ vertebral body was then performed to clarify the diagnosis. Finally, the in-situ hybridization of the tumor of the lumbar vertebrae suggested a non-HIV/transplantation-related EBV-SMT. Consequently, the patient received surgery without chemotherapy and radiotherapy, after which her conditions improved.

Keywords: epstein barr virus, smooth muscle tumor, juvenile idiopathic arthritis, children, hip joint

\section{BACKGROUND}

Epstein-Barr virus-associated smooth muscle tumor (EBV-SMT) is an immunosuppression-related rare tumor that usually occurs in patients with acquired immune deficiency syndrome (AIDS), organ transplantation, or congenital immunodeficiency. Cases with EBV-SMT combined with juvenile idiopathic arthritis (JIA) have not been reported thus far. Herein, we report a single case of EBV-SMT combined with JIA in a 6-year-old patient without HIV, organ transplantation, or congenital immunodeficiency.

\section{CASE PRESENTATION}

A 6-month-old girl presented to our clinic with bilateral hip joint pain, which began 1 year prior to her admission. The pain drastically affected her physical activity and was more evident in the morning. No other symptoms were presented. She then received Plain MRI and enhanced scanning of the hip joints, which revealed a small amount of effusion within the bilateral joints. Both colored 
ultrasound examination of retroperitoneum and Plain CT scanning of the chest and abdomen showed no evident abnormalities; thus, no special treatment was given to the child at that time.

The child still complained of recurrent hip pain, and her parents treated her with physical therapy, with no significant improvement. In September 2019, the girl was admitted to our clinic for the first time, and the Plain MRI and enhanced scanning showed a small amount of bilateral hip joint effusion. Cytological examinations of the bone marrow were normal. However, her parents treated the child with irregular traditional Chinese medicine and still did not see any significant improvement.

In December 2019, the girl was admitted to another hospital and diagnosed with JIA. At that time, EBV-DNA quantification was normal. From then on, she received oral administration of methotrexate (MTX, $14 \mathrm{mg} / \mathrm{m}^{2}$, once per week), methylprednisolone pills $(0.75 \mathrm{mg} / \mathrm{kg} / \mathrm{d})$, and subcutaneous injections of Recombinant Human Tumor Necrosis Factor- $\alpha$ Receptor II; lgG Fc Fusion Protein (0.8 $\mathrm{mg} / \mathrm{kg}$, once per week). The pain was relieved after 4 weeks of continuous treatment. Two months later, the child did not follow-up and stopped all treatment. On May 12, 2020, the pain in the bilateral hip joints became more severe and was accompanied by occasional headaches. The patient was then admitted to our hospital on May 14 for further examinations. No other symptoms were present (Figure 1).

The girl was diagnosed with immune thrombocytopenia (PLT: $87 \times 109 / \mathrm{L})$ in 2017. At that time, cytological examination of the bone marrow showed active bone marrow hyperplasia, maturation disorders of megakaryocytes, and diffused distribution of platelets. The platelet count returned to a normal level after venous infusion of immunoglobulin. No history of organ transplantation or repeated infection was reported. In addition, no remarkable family history was reported.

Upon physical examination, her vital signs were in a normal range with a body weight of $16 \mathrm{~kg}$. The examination of her chest and abdomen showed no obvious abnormalities. No swelling or pressing pain was found in the joints of limbs. However, the 4-shaped sign showed positive results.

During routine laboratory and blood examinations: C-reactive protein (CRP), IL-6, serum ferritin, ASO, and rheumatoid factors (RF) were all within a normal range. Autoimmune antibody (ANCA, ANA, ANA spectrum, PR3-IgG, MPO-IgG, GBMIgG, and anti-CCP) and pathogenic examination (tuberculosis antibody, tuberculosis infection-specific $\mathrm{T}$ cells, mycoplasma antibody, HIV, and fungi) were all negative. EB-VCA-IgG and EB-NA-IgG levels were elevated; EB-VCA-IgM and EB-EA-IgG levels were normal. EBV-DNA quantification showed normal results. Examination of $\mathrm{HBsAg}, \mathrm{HBeAg}$, and $\mathrm{HBcAb}$ showed positive, and serum quantification of HBV-DNA was $3.38 \times$ $10^{7} \mathrm{IU} / \mathrm{ml}$. The levels of immunoglobulins (A, E, G, and M) and complements $\mathrm{C} 3$ and $\mathrm{C} 4$ were all within a normal range (Table 1). Lymphocyte subsets were normal.

Colored ultrasound examination of the retroperitoneum showed no masses or abnormalities. Plain CT scanning of the lungs showed multiple nodules and stripe-shaped shadows bilaterally in the lungs. The largest was $0.63 \times 0.74 \mathrm{~cm}$. Plain and enhanced MRI scanning of the knee and hip joints revealed

\section{The patient came to the hospital with hip-joint pain and obviously in morning.

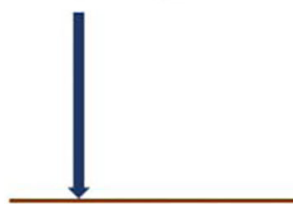 \\ Methotrexate, methylprednisolone, Recombinant Human Tumor Necrosis Factor- $\alpha$ Receptor II.
Hip-joint pain was better than before.

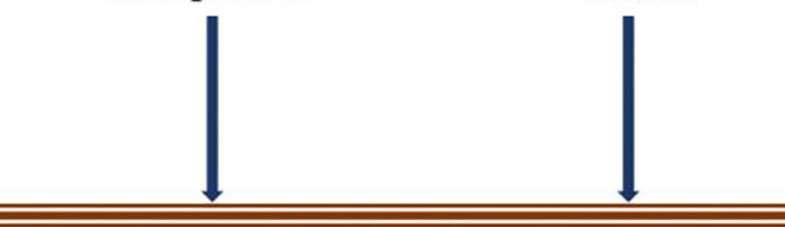
Stopped medicine due to the COVID-19 epidemic. \\ 2020.1 \\ 2020.2 \\ 2020.5 \\ Hip-joint pain increased and occasional headache. \\ 2019.5 \\ 2019.12

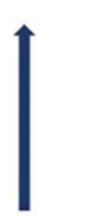 \\ JIA was diagnosed. \\ EBV-SMT was diagnosed.}

FIGURE 1 | Diagram of the disease progression. 
TABLE 1 | Laboratory examination results after hospitalization.

\begin{tabular}{|c|c|c|c|}
\hline Blood routine test & & Anti-nuclear antibodies (ANAs) & Negative \\
\hline White blood cell $\left(\times 10^{9} / \mathrm{L}\right)$ & 5.45 & ANCA & Negative \\
\hline Neutrophils ratio (\%) & 0.593 & PR3-IgG & Negative \\
\hline Lymphocyte ratio (\%) & 0.338 & MPO-IgG & Negative \\
\hline Hemoglobin (g/L) & 131 & GBM-lgG & Negative \\
\hline Platelet $\left(\times 10^{9} / \mathrm{L}\right)$ & 109 & PPD-IgG/lgM & Negative \\
\hline Red blood cell (×10 $12 / \mathrm{L})$ & 4.55 & SPOT-TB test & Negative \\
\hline AST (IU/L) & 26.1 & $\mathrm{Mp}-\mathrm{Ab}$ & Negative \\
\hline $\operatorname{ALT}(I U / L)$ & 23.3 & $\mathrm{HIV}-\mathrm{Ab}$ & Negative \\
\hline Creatinine $(\mu \mathrm{mol} / \mathrm{L})$ & 21.6 & HBsAg & Positive \\
\hline Urea nitrogen (mmol/L) & 3.52 & $\mathrm{HBeAg}$ & Positive \\
\hline CK (U/L) & 69.3 & $\mathrm{HBcAb}$ & Positive \\
\hline CK-MB (IU/L) & 31.7 & HBV-DNA (0-100 IU/ml) & $3.38 \times 10^{7} / \mathrm{L}$ \\
\hline LDH (IU/L) & 229.0 & EB-VCA-lgG (0-20 U/ml) & $>750$ \\
\hline C-reactive protein (0-8 mg/L) & 2.51 & EB-NA-lgG (0-40 U/ml) & $<10$ \\
\hline $\mathrm{IL}-6(<7 \mathrm{pg} / \mathrm{mL})$ & 1.74 & EB-VCA-lgM (0-40 U/ml) & 35.6 \\
\hline Serum ferritin (15-152 ng/mL) & 25.4 & EB-EA-IgG (0-20U/ml) & 48.8 \\
\hline ASO (0-100 IU/ml) & $<25$ & EBV-DNA (0-400 Copies/mL) & $<4 \times 10^{2}$ \\
\hline Anti-CCP (0-5 U/ml) & $<0.50$ & Immunoglobulin A (0.14-1.38 g/L) & 0.57 \\
\hline Rheumatoid factor (0-20 IU/ml) & $<20$ & Immunoglobulin E (<90 IU/ml) & $<5$ \\
\hline Galactomannan (<0.5) & 0.152 & Immunoglobulin G (3.6-10.6 g/L) & 7.87 \\
\hline$(1,3)-\beta$-D-glucan $(0-100.5$ pg/ml) & 8.2 & Immunoglobulin M (0.38-1.44 g/L) & 0.40 \\
\hline AFP (0-9 ng/ml) & 0.99 & C3 complement (0.79-1.52 g/L) & 0.74 \\
\hline CEA (0-10 ng/ml) & 0.49 & C4 complement (0.16-0.38 g/L) & 0.26 \\
\hline
\end{tabular}

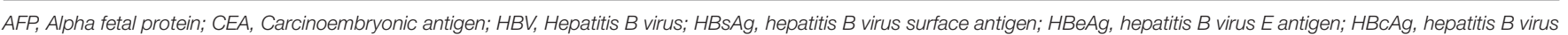

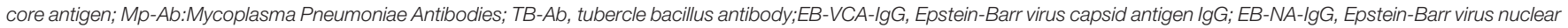

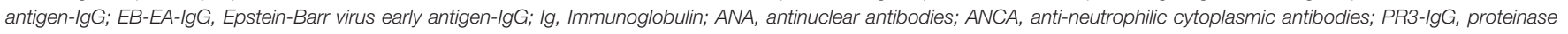
3-lgG; MPO-lgG, myeloperoxidase-lgG; GBM-lgG, glomerular basal membrane-lgG.

that the synovium of the bilateral knee and hip joints were slightly thickened and enhanced, with a small amount of effusion in the joints. Plain and enhanced CT scanning of the lumbar vertebrae showed the adnexa area left to the L2 vertebral body had osteolytic bone destruction and was accompanied by soft tissue masses. Plain and enhanced MRI scanning of the skull, pituitary, and spine showed nodular lesions at the parasellar and meninges of the right parietal lobe and bone mass destruction of the L2 vertebral body and left vertebral pedicle (Figure 2). Electromyography (EMG) of bilateral lower limbs showed no abnormalities in nerve conduction or muscle contraction.

Bone marrow biopsy examination showed active bone marrow hyperplasia, substantially reduced erythroid hyperplasia, and elevated lymphocytes. Biopsy of the inguinal lymphocytes revealed that the microscopy and immunohistochemistry findings agreed with the changes of reactive hyperplasia of the lymph node.

So far, the diagnosis of the child is still not clear. Two biopsies were performed within the L2 vertebral lesion tissues. The first biopsy was not diagnosed because EBER in-situ hybridization was not performed. The diagnosis was not made until after the second biopsy of a positive EBER in-situ hybridization was performed (Figure 3).

The girl's pathological examination showed EBER (+); thus, she was diagnosed with EBV-SMT. However, she was not previously infected with HIV or had any organ transplantation. In addition, the girl had no history of immunodeficiencyrelated diseases. In order to clarify the possibility of undiagnosed immunodeficiency or other causes, blood was obtained for whole-exome sequencing. The results showed no related pathogenic genes.

The patient was admitted to the hospital for arthritis. The imaging examinations after admission showed multiple lesions in the skull, lungs, and vertebral body. Other hematological tumors or Langerhans cell histiocytosis were considered during the diagnosis, which was not in agreement with the pathological and other examination results. Biopsy of the L2 vertebral body was then performed to clarify the diagnosis. Finally, the in-situ hybridization of the tumor of the lumbar vertebrae suggested non-HIV/transplantation-related EBV-SMT. The patient then received surgery (lumbar lesions resection + fusion bone grafting and internal fixation) without chemo or radiotherapy. The hip pain improved except for occasional headache. The patient is still under regular follow-up now.

\section{DISCUSSION}

SMT was first reported by Pritzker et al. (1) in 1970 in a patient who underwent organ transplantation. However, the causal relationship with EBV was not verified until 1995 (2). To 
A
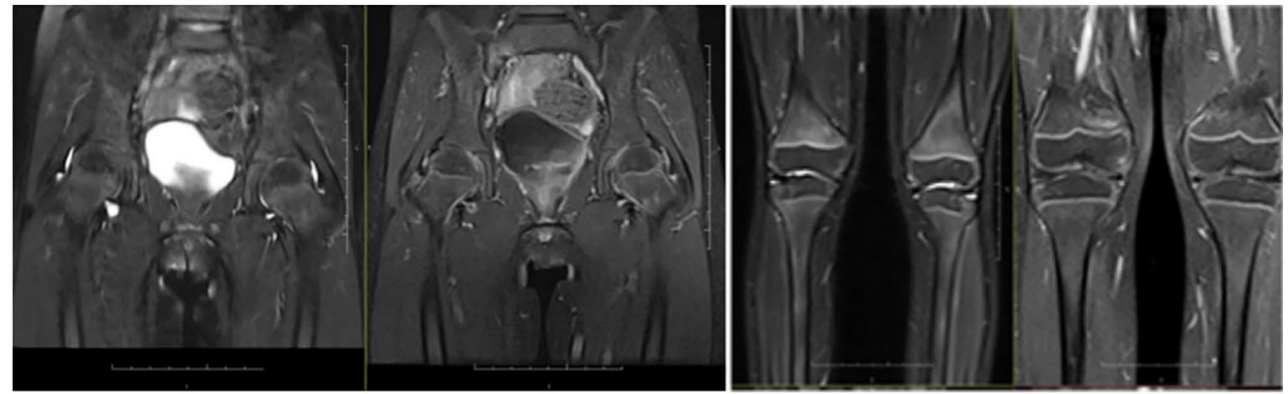

B

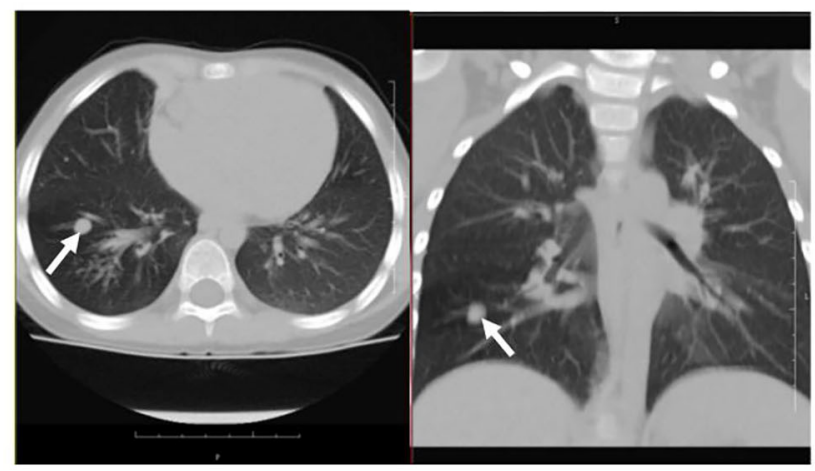

C

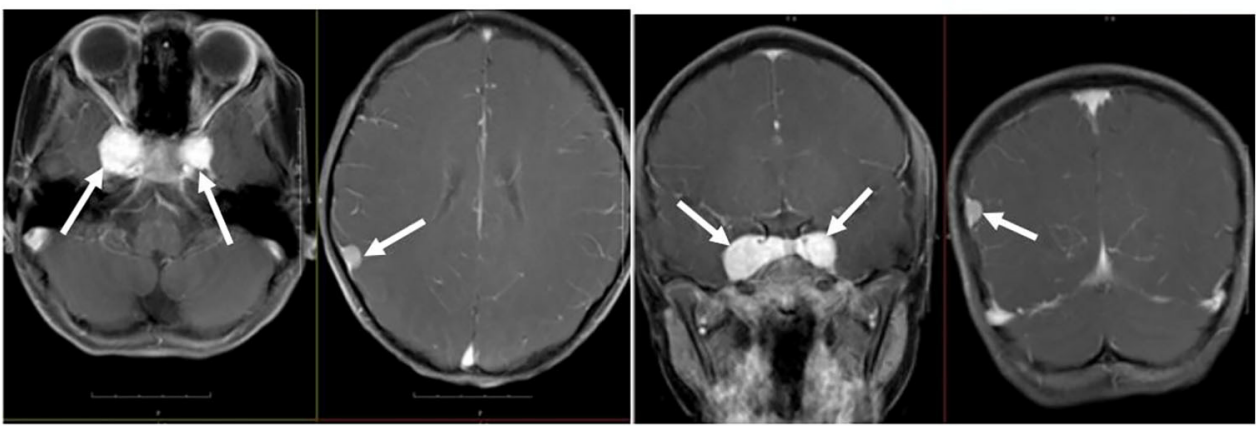

D

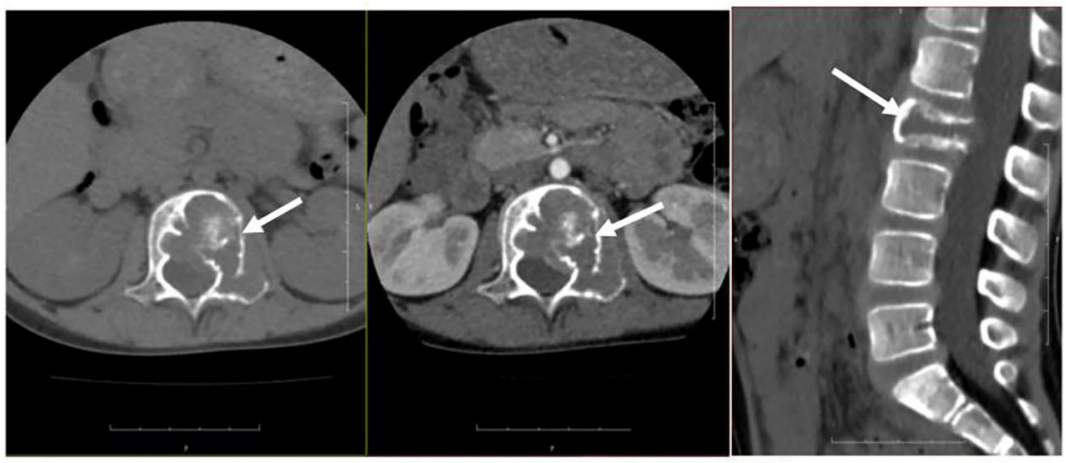

FIGURE 2 | Imaging examination. (A) Plain and enhanced MRI scanning of the knee joints and hip joints showed that the synovium of bilateral knee and hip joints were slightly thickened and enhanced, with a small amount of effusion in joints. (B) Plain CT scanning of lungs showed multiple nodules and stripe-shaped shadows in bilateral lungs, and the size of the largest shadow was $0.63 \times 0.74 \mathrm{~cm}$. (C) Plain and enhanced MRI scanning of the skull showed nodular lesions at the parasellar and meninges of the right parietal lobe. (D) Plain and enhanced MRI scanning of the spine showed bone mass destruction of the L2 vertebral body and left vertebral pedicle. 

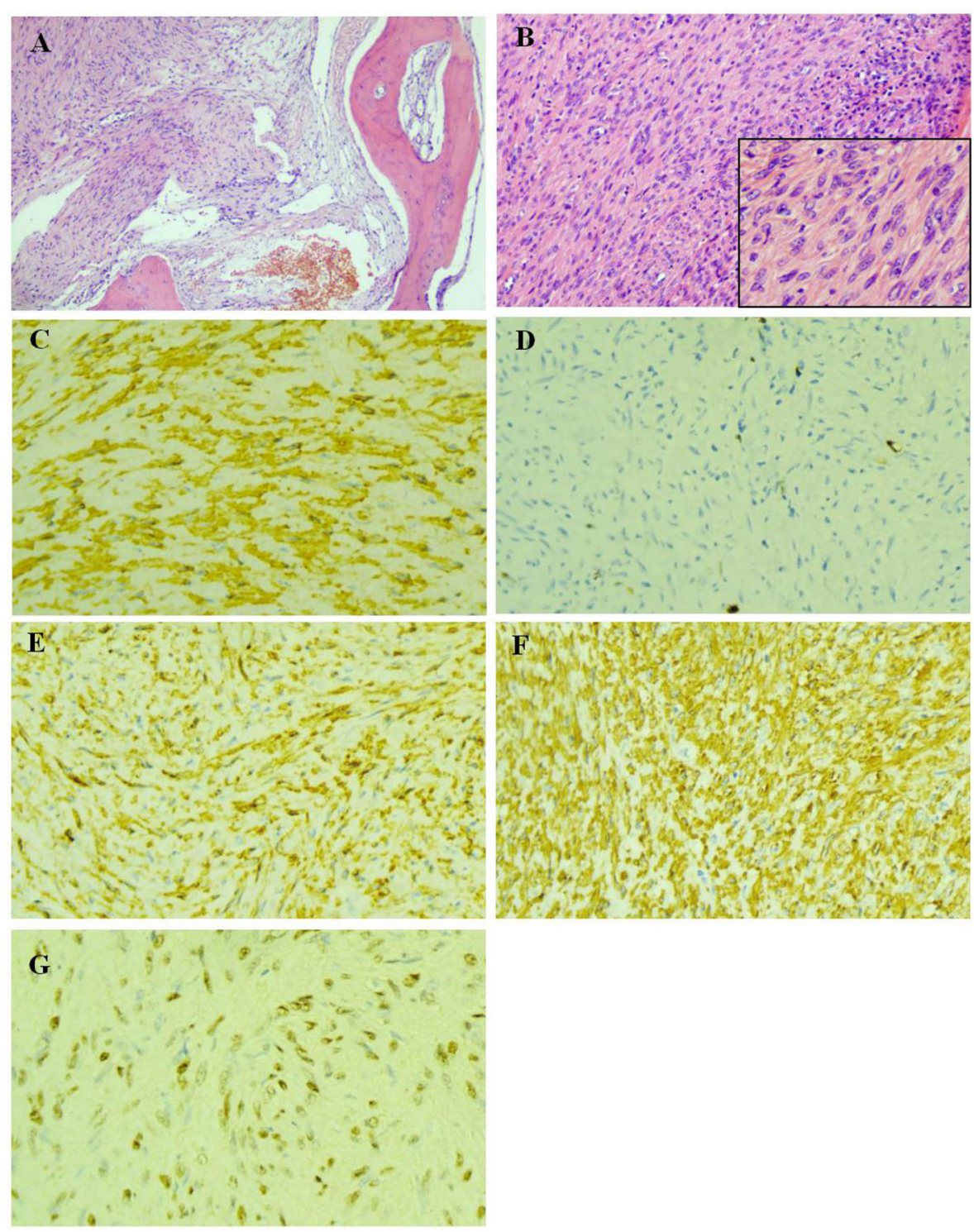

FIGURE 3 | Pathological examination of the biopsy of lesion tissues of the L2 vertebral body. (A) The bone tissue were invaded by tumor cells (HE $\times 100)$. (B) The tumor cells was spindle-shaped, with abundant cytoplasm, red stain and mild atypia, while no evident nuclear fission was observed (HE $\times 200)$. (C) The actin were expressed in the cytoplasm of tumor cells (IHC $\times$ 400). (D) The highest ki-67 positive index was $6 \%(I H C \times 400)$. (E) The $h$-Caldesmon were expressed diffusely in the cytoplasm of tumor cells $(\mathrm{IHC} \times 400)$. (F) SMA were expressed diffusely in the cytoplasm of tumor cells $(\mathrm{IHC} \times 400)$. (G) Epstein-Barr virus encoded RNA situ hybridization showed diffusely positive (EBER $\times$ 400).

date, studies have demonstrated that patients sensitive to EBVSMT include those with HIV infection, patients who underwent immunosuppressive therapy after organ transplantation, and those with congenital immunodeficiency (3-5). Although, EBVSMT can occur in both children and adults, simple SMT in children is exceedingly rare. However, with the prevalence of AID, the occurrence of HIV-SMT in children has increased.

EBV-SMT has become the second leading tumor in AID afflicted children (6). However, the incidence rate of EBV-SMT is still unclear. In a study performed by Stubbins et al. (7) in 2019, 474 children and 4532 adults received organ transplantation at the University of Alberta Hospital between January 1984 and December 2015, and only three developed EBV-SMT. Another study reviewed 975 adult patients who received renal transplantation between 1985 and 2000 and revealed that 16 patients developed EBV-SMT, with an overall incidence of 1.67\% (8). Nevertheless, previous studies were all single-center studies of single disease types. So far, no large-scale study has been performed to investigate the overall incidence of the disease.

With an increasing understanding of EBV-SMT, more and more cases of EBV-SMT have been reported. However, most cases were immunocompromised patients, while EBV-SMT patients 
with autoimmune diseases have been rarely reported. In 2019, So et al. (9) reported a case of a 49-year-old female patient with idiopathic inflammatory myopathy, which was also the first reported case of an autoimmune disease accompanied by EBV-SMT. In this study, we reported the first case of a patient with JIA associated with EBV-SMT. Unlike previously reported cases, our patient was incredibly young and received short-term immunosuppressive therapy; the EBV-SMT occurred secondary to JIA. However, it remains unclear whether EBV-SMT was associated with the application of an immunosuppressant agent, the state of JIA, or a dormant EBV activation after infection. Interestingly, $\mathrm{Au}$ et al. (10) found that treating autoimmune diseases with azathioprine or cyclophosphamide could induce EBV-negative lymphoproliferative disorders, suggesting that drugs could directly induce lymphoproliferative disorders. On the other hand, Wang et al. (11) reported a 31-year-old nonHIV/organ transplantation/immunodeficiency patient who was pathologically confirmed with multiple intestinal EBV-SMT. Their data demonstrated that EBV-SMT could also occur in patients with normal immune functions (11). The above findings demonstrated that the pathogeneses of EBV-SMT involve multiple pathways and mechanisms.

The exact pathogenesis of EBV-SMT is still unclear. Currently, it is well-known that EBV infection is closely associated with immune suppression. For instance, negative serum EBV before transplantation, idiopathic EBV infection after transplantation, and high viral load have been identified as independent risk factors of post-transplantation EBV-SMT (7). In addition, more potent immunosuppression, such as thymectomy, could also be a risk factor inducing EBV-SMT (7). However, the exact mechanisms involved in the invasion of EBV into smooth muscle cells and the consequent proliferation and transformation remain poorly understood. With the advancement of EBV-SMT research over recent years, several studies reported that the pathogeneses of EBV-SMT could be associated with the infection of smooth muscle cells and a tumor transformation induced by cloning and amplification of EBV (12). Nonetheless, the invasion of EBV into smooth muscle cells is still not fully understood. Previous studies showed a positive expression of EBV receptor CD21 receptor in patients with HIV-SMT, suggesting that EBV could invade smooth muscle cells via this pathway (13). However, the CD21 receptor staining of the SMT patient after transplantation was negative, suggesting that there are other pathways involved in SMT occurrence. For instance, the infection could occur by fusing smooth muscle cells with the EBV-infected lymphocytes, which needs to be further investigated $(2,14)$. Still, the transformation and proliferation of EBV after invading smooth muscle are of great importance and should be addressed by future studies.

In conclusion, EBV-SMT is a rare tumor lacking specificity in clinical practices; thus, it could be easily misdiagnosed as

\section{REFERENCES}

1. Pritzker KP, Huang SN, Marshall KG. Malignant tumours following immunosuppressive therapy. Can Med Assoc J. (1970) 103:1362-5. other tumorous diseases. The diagnosis of EBV-SMT relies on pathological examinations and EBER in-situ hybridization, while tumor resection and discontinuation of immunosuppression are still the main treatment approaches for this disease. Herein, we reported a rare case that suggested how EBV-SMT can occur secondary to immune diseases not only in patients with AID, after organ transplantation, or congenital immunodeficiency. Unfortunately, whether the occurrence of EBV-SMT in this case was related to the application of immunosuppressant agent, the state of JIA, or a dormant EBV activation after infection is unknown. The interaction among them needs further study.

\section{DATA AVAILABILITY STATEMENT}

The original contributions presented in the study are included in the article/supplementary material, further inquiries can be directed to the corresponding author/s.

\section{ETHICS STATEMENT}

The studies involving human participants were reviewed and approved by the Institutional Review Board of the Hunan Children's Hospital. Written informed consent to participate in this study was provided by the participants' legal guardian/next of kin. Written informed consent was obtained from the minor(s)' legal guardian for the publication of any potentially identifiable images or data included in this article.

\section{AUTHOR CONTRIBUTIONS}

ZK, JX, and ZL contributed to conception and design of the study. JX organized the data. ZK wrote the first draft of the manuscript. ZL reviewed sections of the manuscript. All authors contributed to manuscript revision, read, and approved the submitted version.

\section{FUNDING}

This work was supported by the Key Research and Development Program of Hunan Province in China (2018SK2139) and Clinical Center for Acute Renal Injury in Children of Hunan Province (2016SK4004).

\section{ACKNOWLEDGMENTS}

The authors thank all colleagues who processed medical records, managed patient and assisted with follow-up. We would like to express our gratitude to the patient and their family members, who made this study possible. The authors thank AiMi Academic Services (www.aimieditor.com) for English language editing and review services.

2. Lee ES, Locker J, Nalesnik M, Reyes J, Jaffe R, Alashari M, et al The association of Epstein-Barr virus with smooth-muscle tumors occurring after organ transplantation. N Engl J Med. (1995) 332:19-25. doi: 10.1056/NEJM199501053320104 
3. Cohen JI. GATA2 deficiency and Epstein-Barr virus disease. Front Immunol. (2017) 8:1869. doi: 10.3389/fimmu.2017.01869

4. Dekate J, Chetty R. Epstein-Barr virus-associated smooth muscle tumor. Arch Pathol Lab Med. (2016) 140:718-22. doi: 10.5858/arpa.2015-0120-RS

5. Conrad A, Brunet AS, Hervieu V, Chauvet C, Buron F, Collardeau-Frachon $\mathrm{S}$, et al. Epstein-Barr virus-associated smooth muscle tumors in a composite tissue allograft and a pediatric liver transplant recipient. Transpl Infect Dis. (2013) 15:E182-6. doi: 10.1111/tid.12126

6. Gupta S, Havens PL, Southern JF, Firat SY, Jogal SS. EpsteinBarr virus-associated intracranial leiomyosarcoma in an HIVpositive adolescent. J Pediatr Hematol Oncol. (2010) 32:e144-7. doi: 10.1097/MPH.0b013e3181c80bf3

7. Stubbins RJ, Alami Laroussi N, Peters AC, Urschel S, Dicke F, Lai RL, et al. Epstein-Barr virus associated smooth muscle tumors in solid organ transplant recipients: incidence over 31 years at a single institution and review of the literature. Transpl Infect Dis. (2019) 21:e13010. doi: 10.1111/tid.13010

8. Tan CS, Loh HL, Foo MW, Choong LH, Wong KS, Kee TY. EpsteinBarr virus-associated smooth muscle tumors after kidney transplantation: treatment and outcomes in a single center. Clin Transplant. (2013) 27:E462-8. doi: $10.1111 /$ ctr.12139

9. So H, Wong VTL, Pang HT, Lao VWN, Yip RML. The first case of idiopathic inflammatory myopathy complicated by Epstein-Barr virus-associated smooth muscle tumor and lymphoma. Int J Rheum Dis. (2019) 22:1342-3. doi: 10.1111/1756-185X.13619

10. Au WY, Ma ES, Choy C, Chung LP, Fung TK, Liang R, et al. Therapyrelated lymphomas in patients with autoimmune diseases after treatment with disease-modifying anti-rheumatic drugs. Am J Hematol. (2006) 81:5-11. doi: $10.1002 /$ ajh. 20508

11. Wang R, Liao XH, Li SH, Liu DW. [Mutifocal EB virus associated smooth muscle tumor arising in intestinal tract of non-transplantation/HIV patient: report of a case]. Zhonghua Bing Li Xue Za Zhi. (2020) 49:938-40. doi: 10.3760/cma.j.cn112151-20191226-00829

12. Purgina B, Rao UN, Miettinen M, Pantanowitz L. AIDS-related EBVassociated smooth muscle tumors: a review of 64 published cases. Patholog Res Int. (2011) 2011:561548. doi: 10.4061/2011/561548

13. Jossen J, Chu J, Hotchkiss H, Wistinghausen B, Iyer K, Magid M, et al. Epstein-Barr virus-associated smooth muscle tumors in children following solid organ transplantation: a review. Pediatr Transplant. (2015) 19:235-43. doi: $10.1111 /$ petr.12426

14. Bayliss GJ, Wolf H. Epstein-Barr virus-induced cell fusion. Nature. (1980) 287:164-5. doi: $10.1038 / 287164 \mathrm{a} 0$

Conflict of Interest: The authors declare that the research was conducted in the absence of any commercial or financial relationships that could be construed as a potential conflict of interest.

Copyright (๑) 2021 Kang, Xu and Li. This is an open-access article distributed under the terms of the Creative Commons Attribution License (CC BY). The use, distribution or reproduction in other forums is permitted, provided the original author(s) and the copyright owner(s) are credited and that the original publication in this journal is cited, in accordance with accepted academic practice. No use, distribution or reproduction is permitted which does not comply with these terms. 